\title{
Comparisons of Distributed Energy Efficient Clustering (DEEC) and its variation for WSN to the Internet of Things Applications
}

\author{
Hamdy H. El-Sayed
}

Faculty of Computers and Artificial Intelligence, Department of Computer Science, Sohag University, Sohag, 82524, Egypt

Email: Hamdy2006x@gmail.com

Zainab Mostafa Hashem

Faculty of Science, Department of Computer Science, Sohag University, Sohag, 82524, Egypt

Email: mostafazainab923@gmail.com

ABSTRACT-

The main thing we work on is Wireless Sensor Networks (WSNs) which contain numerous sensor nodes with limited power resources, which report sensed data to the Base Station (BS) that requires high energy usage. We want to let the battery live as long as we can, as the cost of changing batteries of nodes is high and also difficult. So, we use efficient protocols which improve the way the sensors use to send and receive sensed data to BS on other hand, nodes closer to the base station are exploited as they have to spend additional energy in relying on data of faraway nodes. This brings in the idea of implementing blanket coverage in heterogeneous wireless sensor networks for the internet of things. In this paper, we first test Distributed Energy Efficient Clustering (DEEC), Developed DEEC (DDEEC), Enhanced DEEC (EDEEC), Threshold DEEC (TDEEC), and Improved DEEC Protocol (IDEEC) under several different scenarios. We observe thoroughly the performance based on stability period, network lifetime, and throughput. EDEEC and TDEEC perform better in all heterogeneous scenarios containing variable heterogeneity in terms of lifetime, however, TDEEC is best of all for the stability period of the network. IDEEC is better than DDEEC in terms of Overhead, but TDEEC is the best. However, the performance of DEEC and DDEEC is highly affected by changing the heterogeneity parameters of the network.

Keywords - Cluster, Head, Residual, Energy, Efficient, Wireless, Sensor, Networks, Heterogeneous network, Stability period, Initial energy, IoT, DEEC, DDEEC, EDEEC, IDEEC,TDEEC.

\section{INTRODUCTION}

W SNs are extremely valuable in a variety of essential applications, including military surveillance, environmental, traffic, temperature, pressure, vibration monitoring, and disaster relief. to obtain fault tolerance, WSN is made up of hundreds or even thousands of nodes. a set of sensors placed at random throughout the study area [1]. All nodes must send their data to BS, which is also known as "sink". Usually, nodes in WSN are power constrained due to limited battery, it is also not possible to recharge or replace the battery of already deployed nodes and nodes might be placed where they cannot be accessed. Since nodes may be located far from the BS, direct communication is not possible due to battery limitations. Direct communication consumes a lot of energy. Clustering is a significant strategy for reducing battery consumption in which cluster members choose a Cluster Head $(\mathrm{CH})$. In this regard, many clustering protocols have been proposed $[2,3]$.

All cluster nodes transmit their data to $\mathrm{CH}$, which aggregates the data and provides the aggregated data to $\mathrm{BS}$ $[4,6]$. Fewer messages are delivered to BS with aggregation, and just a few nodes must broadcast over long distances, saving energy and extending the network's overall lifetime.
When compared to data transmission, energy usage for data aggregation is substantially lower. Clustering can be done on homogeneous and heterogeneous networks, respectively. Homogeneous networks have nodes with the same energy level, while heterogeneous networks have nodes with variable energy levels.

Low-Energy Adaptive Clustering Hierarchy (LEACH) [5], Power-Efficient Gathering in Sensor Information Systems (PEGASIS) [7], and Hybrid Energy-Efficient Distributed Clustering (HEED) [8] are algorithms designed for homogeneous WSNs, so these protocols do not work efficiently in heterogeneous scenarios because these algorithms are unable to treat nodes differently in terms of their energy. Stable Election Protocol (SEP) [9], Distributed Energy-Efficient Clustering (DEEC) [10], Developed DEEC (DDEEC) [11,14], Enhanced DEEC (EDEEC) [12], Threshold DEEC (TDEEC) [13], and Improved DEEC (IDEEC) [15,16] are heterogeneous WSN algorithms. Because SEP is developed for two-level heterogeneous networks, it will not perform well in threelevel or multilevel heterogeneous networks. Only normal and advanced nodes are considered in SEP, with normal nodes having low energy and advanced nodes having high energy. DEEC, DDEEC, EDEEC, TDEEC, and IDEEC are multilevel heterogeneous network algorithms that can also perform well in two-level heterogeneous scenarios. 
In this paper, we study the performance of heterogeneous WSN protocols under multi-level heterogeneous networks. We compare the performance of DEEC, DDEEC, EDEEC, TDEEC, and IDEEC for different scenarios of multilevel heterogeneous WSNs.

We discriminate each protocol based on prolonging stability period, a network lifetime of nodes alive during rounds for heterogeneous networks, delay, overhead, packet delivery ratio, throughput.

It is found that different protocols have different efficiency for three-level and multilevel heterogeneous WSNs in terms of stability period, nodes alive and network lifetime. DEEC, DDEEC, and IDEEC perform well under threelevel heterogeneous WSNs in terms of the stability period. However, it lacks in performance as compared to EDEEC and TDEEC in terms of network lifetime. Whereas, EDEEC and TDEEC perform well under multi and threelevel heterogeneous WSNs containing low energy level difference between normal, advanced, and super nodes in terms of both stability period and network lifetime.

\section{RELATED WORK}

Heinzeman, et al. [5] proposed LEACH, a clustering method for homogeneous WSNs in which nodes choose to be $\mathrm{CHs}$ at random and send this selection criterion throughout the network to spread energy load. G. Smaragdakis et al. [9] created the SEP protocol, in which each sensor node in a heterogeneous two-level hierarchical network elects itself as a $\mathrm{CH}$ based on its starting energy compared to other nodes. L. Qing, Q. Zhu, and M. Wang [10] studied heterogeneous WSNs and presented the DEEC protocol, in which $\mathrm{CH}$ selection is based on the probability of the network's residual and average energy ratio. Brahim Elbhiri, et al. [11] researched heterogeneous WSNs and presented the DDEEC protocol, which is based on residual energy for $\mathrm{CH}$ selection and network balancing. As a result, advanced nodes are more likely to be chosen as $\mathrm{CH}$ during the initial transmission rounds, but as their energy decreases, they will have the same $\mathrm{CH}$ election chance as regular nodes. P. Saini et al. [12] introduced the EDEEC protocol, which is expanded to three levels of heterogeneity by introducing a new energy level called super nodes. Parul Saini and Ajay K Sharma [13] created the TDEEC method, which chooses the $\mathrm{CH}$ from high-energy nodes, enhancing network energy efficiency and lifetime. Xie [14] proposed an improved distributed energy-saving clustering algorithm (IDEEC) for HWSNs in 2017. IDEEC takes into account the multilevel energy model, simplifies the threshold, improves the probability of cluster head selection, and optimizes the average energy in the network.

\section{OVERVIEW OF DISTRIBUTED HETEROGENOUS PROTOCOLS}

\section{$1.1 \quad$ DEEC}

DEEC is designed to deal with nodes of heterogeneous WSNs. For $\mathrm{CH}$ selection, DEEC uses the initial and residual energy levels of nodes. Let $n_{i}$ denote the number of rounds to be a for the node $s_{i} \cdot p_{o p t} N$ is the optimum number of in our network during each round. Selection criteria in DEEC are based on the energy level of nodes. As in a homogenous network, when nodes have the same amount of energy during each epoch then choosing $\mathrm{p}_{\mathrm{i}}=$ $\mathrm{p}_{\text {opt }}$ assures that $\mathrm{CHs}$ during each round. In WSNs, nodes with high energy are more probable to become than nodes with low energy but the net value of $\mathrm{CHs}$ during each round is equal to $p_{o p t} N$. $p_{i}$ is the probability for each node to become, so, a node with high energy has a larger value $\operatorname{asp}_{\mathrm{i}}$ compared to the $\mathrm{p}_{\mathrm{opt}} \cdot \overline{\mathrm{E}}(\mathrm{r})$ denotes average energy of network during the round $r$ which can be given as in [10].

$$
\bar{E}(r)=\frac{1}{N} \sum_{i=1}^{N} E_{i}(r)
$$

Probability for $\mathrm{CH}$ selection in DEEC is given as in [10] :

$$
\begin{array}{r}
p_{i}=p_{o p t}\left[1-\frac{\bar{E}(r)-E_{i}(r)}{\bar{E}(r)}\right] \\
=p_{o p t} \frac{E_{i}(r)}{\bar{E}(r)}
\end{array}
$$

In DEEC the average total number of $\mathrm{CH}$ during each round is given as in [10] :

$$
\begin{gathered}
\sum_{i=1}^{N} p_{i}=\sum_{i=1}^{N} p_{o p t} \frac{E_{i}(r)}{\bar{E}(r)}=p_{o p t} \sum_{i=1}^{N} \frac{E_{i}(r)}{\bar{E}(r)} \\
=N p_{o p t}
\end{gathered}
$$

$\mathrm{p}_{\mathrm{i}}$ is probability of each node to become $\mathrm{CH}$ in a round. Where $\mathrm{G}$ is the set of nodes eligible to become $\mathrm{CH}$ at roundr. If node becomes $\mathrm{CH}$ in recent rounds, then it belongs toG. During each round each node chooses a random number between 0 and 1 . If number is less than threshold as defined in equation 4 as in [10], it is eligible to become a $\mathrm{CH}$ else not.

$$
\mathrm{T}\left(\mathrm{s}_{\mathrm{i}}\right)= \begin{cases}\frac{\mathrm{p}_{\mathrm{i}}}{1-\mathrm{p}_{\mathrm{i}}\left(\operatorname{rmod} \frac{1}{\mathrm{P}_{\mathrm{i}}}\right)} & \text { if } \mathrm{s}_{\mathrm{i}} \in \mathrm{G} \\ 0 & \text { otherwise }\end{cases}
$$

As $p_{o p t}$ is reference value of average probability $p_{i}$. In homogenous networks, all nodes have same initial energy so they use $\mathrm{p}_{\mathrm{opt}}$ to be the reference energy for probability $\mathrm{p}_{\mathrm{i}}$. However, in heterogeneous networks, the value of $\mathrm{p}_{\mathrm{opt}}$ is different according to the initial energy of the node. In two level heterogeneous network the value of $\mathrm{p}_{\mathrm{opt}}$ is given by as in [10]:

$$
\mathrm{p}_{\mathrm{adv}}=\frac{\mathrm{p}_{\mathrm{opt}}}{1+\mathrm{am}}, \mathrm{p}_{\mathrm{nrm}}=\frac{\mathrm{p}_{\mathrm{opt}}(1+\mathrm{a})}{(1+\mathrm{am})}
$$


Then use the above $\mathrm{p}_{\mathrm{adv}}$ and $\mathrm{p}_{\mathrm{nrm}}$ instead of $\mathrm{p}_{\mathrm{opt}}$ in equation 2 for two level heterogeneous network as supposed in [10]:

$$
p_{i}= \begin{cases}\frac{p_{o p t} E_{i}(r)}{(1+a m) E(r)} & \text { if } s_{i} \text { is the normal node } \\ \frac{p_{o p t}(1+a) E_{i}(r)}{(1+a m) E(r)} & \text { if } s_{i} \text { is the advanced node }\end{cases}
$$

Above model can also be extended to multi-level heterogenous network given below as in [10] :

$$
p_{\text {multi }}=\frac{p_{\text {opt }} N\left(1+a_{i}\right)}{\left(N+\sum_{i=1}^{N} a_{i}\right)}
$$

Above $p_{\text {multi }}$ in equation 2 instead of $p_{\text {opt }}$ to get $p_{i}$ for heterogeneous node. $p_{i}$ for the multilevel heterogeneous network is given by as in [10] :

$$
p_{i}=\frac{p_{o p t} N(1+a) E_{i}(r)}{\left(N+\sum_{i=1}^{N} a_{i}\right) \bar{E}(r)}
$$

In DEEC we estimate average energy $E(r)$ of the network for any round $r$ as in $[10]$ :

$$
\overline{\mathrm{E}}(\mathrm{r})=\frac{1}{\mathrm{~N}} \mathrm{E}_{\text {total }}\left(1-\frac{\mathrm{r}}{\mathrm{R}}\right)
$$

$\mathrm{R}$ denotes total rounds of network lifetime and is estimated as follows:

$$
\mathrm{R}=\frac{\mathrm{E}_{\text {total }}}{\mathrm{E}_{\text {round }}}
$$

$E_{\text {total }}$ is total energy of the network where $E_{\text {round }}$ is energy expenditure during each round.

\subsection{DDEEC}

DDEEC [14] implements the same strategy like DEEC in terms of estimating average energy of networks and the cluster head selection algorithm which is based on residual energy where [11]:

- The average energy of $r_{\text {th }}$ round is set at equation (9). Where $\mathrm{R}$ denote the total rounds of the network lifetime and is defined at equation (10).

- $\mathrm{E}_{\text {Round }}$ is the total energy dissipated in the network during a round, is equal to:

$$
\begin{aligned}
& \mathrm{E}_{\text {round }}=\mathrm{L}\left(2 * \mathrm{~N} * \mathrm{E}_{\text {elec }}+\mathrm{N} * \mathrm{E}_{\mathrm{DA}}+\mathrm{K} * \varepsilon \mathrm{mp} *\right. \\
& \left.\mathrm{d}_{\mathrm{toBs}}^{4}+\mathrm{N} * \varepsilon f * \mathrm{~d}_{\mathrm{toCH}}^{2}\right)
\end{aligned}
$$

Where $\mathrm{k}$ is the number of clusters, $E_{D A}$ is the data aggregation cost expended in the cluster heads, $d_{\text {toBs }}$ is theaverage distance between the cluster head and the base station, and $d_{\text {toCH }}$ is the average distance between the cluster members and the cluster head.

- Because we assuming that the nodes are uniformly distributed, we can get:

$\mathrm{d}_{\mathrm{toCH}}=\mathrm{M} / \sqrt{2} \pi \mathrm{k}, \mathrm{d}_{\mathrm{toBS}}=0.765^{*} \mathrm{M} / 2$

- The optimal number of clusters is defined as:

$\mathrm{K}_{\mathrm{opt}}=\frac{\mathrm{M}}{\mathrm{d}^{2} \text { toBs }} \frac{\sqrt{\mathrm{N}}}{\sqrt{2 \pi}} \frac{\sqrt{\mathrm{Ef}_{\mathrm{S}}}}{\sqrt{\mathrm{Emp}}}$

In this way, we continue to punish more just these nodes, so they spent more energy and they will die quickly 1 . To avoid this unbalanced case, our protocol DDEEC introduce some changes on the equation 6 . These changes are based on using a threshold residual energy value $\mathrm{Th}_{\mathrm{REV}}$, which is equal to:

$\mathrm{Th}_{\mathrm{REV}}=\mathrm{E}_{0}\left(1+\frac{\mathrm{aE}_{\mathrm{disNN}}}{\mathrm{E}_{\mathrm{disNN}}-\mathrm{E}_{\mathrm{disAN}}}\right)$

Therefore, the cluster head election will be balanced and more equitable. So, the equation (6) which represents the nodes average probability pi to be a cluster head will change as follows:

$p_{i}= \begin{cases}\frac{p_{o p t} E_{i}(r)}{(1+a m) E(r)} & \text { for Nml nodes, } E_{i}(r)>T h_{R E V} \\ \frac{(1+a) p_{o p t} E_{i}(r)}{(1+a m) E(r)} & \text { for Adv nodes, } E_{i}(r)>T h_{R E V} \\ c \frac{(1+a) p_{o p t} E_{i}(r)}{(1+a m) \bar{E}(r)} & \text { for Adv, Nml nodes, } E_{i}(r) \leq T h_{R E V}\end{cases}$

The value of $T h_{\mathrm{REV}}$ is written as $\mathrm{Th} h_{\mathrm{REV}}=\mathrm{b}_{\mathrm{Eo}}$ where

$\mathrm{b}=\left(1+\frac{a \mathrm{E}_{\mathrm{disNN}}}{\mathrm{E}_{\mathrm{disNN}}-\mathrm{E}_{\mathrm{disAN}}}\right)$

\subsection{EDEEC}

E-DEEC implements the same strategy for estimating the energy in the network as proposed in DEEC[10]. Since the probabilities calculated depend on the average energy of the network at round $r$, hence this is to be calculated. This average energy is estimated in equation (9).Where $\mathrm{R}$ denotes the total rounds of the network lifetime. $\mathrm{R}$ can be calculated in equation (10). $\mathrm{d}_{\mathrm{toBS}} \& \mathrm{~d}_{\mathrm{toCH}}$ is calculated in equation (12). During each round, node decide whether to become a $\mathrm{CH}$ or not based on threshold calculated by suggested percentage of $\mathrm{CH}$ and the number of times the node has been a $\mathrm{CH}$ so far. This decision is taken by nodes by choosing a random number between $0 \& 1$. If number is less than threshold $\mathrm{T}(\mathrm{s})$, the node become a $\mathrm{CH}$ for current round. Threshold is calculated as:

$T(s)= \begin{cases}\frac{p}{1-p\left(\operatorname{rmod} \frac{1}{p}\right)} & \text { if } s \in G \\ 0 & \text { otherwise }\end{cases}$ 
where $p, r$, and $G$ represent, respectively, the desired percentage of cluster-heads, the current round number, and the set of nodes that have not been cluster-heads in the last $1 / p$ rounds. Using this threshold, each node will be a cluster head, just once at some point within $1 / p$ rounds. In the three level heterogeneous networks there are three types of nodes normal nodes, advanced nodes and super nodes, based on their initial energy. Hence the reference value of $p$ is different for these types of nodes. The probabilities of normal, advanced and super nodes are [12]:

$$
p_{i}= \begin{cases}\frac{p_{o p t} E_{i}(r)}{(1+m \cdot(a+m o \cdot b)) \bar{E}(r)} & \text { if } s_{i} \text { is the normal node } \\ \frac{p_{o p t}(1+a) E_{i}(r)}{(1+m \cdot(a+m o \cdot b)) \bar{E}(r)} & \text { if } s_{i} \text { is the advanced node } \\ \frac{p_{o p t}(1+b) E_{i}(r)}{(1+m \cdot(a+m o \cdot b)) \bar{E}(r)} & \text { if } s_{i} \text { is the super node }\end{cases}
$$

Threshold for cluster head selection is calculated for normal, advanced, super nodes by putting above values in Equation (17)

$\mathrm{T}\left(\mathrm{s}_{\mathrm{i}}\right)= \begin{cases}\frac{\mathrm{p}_{\mathrm{i}}}{1-\mathrm{p}_{\mathrm{i}}\left(\operatorname{rmod} \frac{1}{\mathrm{p}_{\mathrm{i}}}\right)} & \text { if } \mathrm{p}_{\mathrm{i}} \in \mathrm{G}^{\prime} \\ \frac{\mathrm{p}_{\mathrm{i}}}{1-\mathrm{p}_{\mathrm{i}}\left(\operatorname{rmod} \frac{1}{\mathrm{p}_{\mathrm{i}}}\right)} & \text { if } \mathrm{p}_{\mathrm{i}} \in \mathrm{G}^{\prime \prime} \\ \frac{\mathrm{p}_{\mathrm{i}}}{1-\mathrm{p}_{\mathrm{i}}\left(\operatorname{rmod} \frac{1}{\mathrm{p}_{\mathrm{i}}}\right)} & \text { if } \mathrm{p}_{\mathrm{i}} \in \mathrm{G}^{\prime \prime \prime} \\ 0 & \text { otherwise }\end{cases}$

where $\mathrm{G}^{\prime}$ is the set of normal nodes that have not become cluster heads within the last $1 / \mathrm{pi}$ rounds of the epoch where $s_{i}$ is normal node, $G^{\prime \prime}$ is the set of advanced nodes that have not become cluster heads within the last $1 /$ pi rounds of the epoch where $s_{i}$ is advanced node, $G^{\prime \prime}$ ' is the set of super nodes that have not become cluster heads within the last $1 / \mathrm{pi}$ rounds of the epoch where $s_{i}$ is super node [12].

\subsection{TDEEC}

TDEEC uses same mechanism for $\mathrm{CH}$ selection and average energy estimation as proposed in DEEC. At each round, nodes decide whether to become a $\mathrm{CH}$ or not by choosing a random number between 0 and 1 . If number is less than threshold $\mathrm{T}_{\mathrm{S}}$ as shown in equation 20 then nodes decide to become a $\mathrm{CH}$ for the given round. In TDEEC, threshold value is adjusted and based upon that value a node decides whether to become a $\mathrm{CH}$ or not by introducing residual energy and average energy of that round with respect to optimum number of $\mathrm{CHs}$ [13]. Threshold value proposed by TDEEC is given as follows as in [13]:

$$
\mathrm{T}(\mathrm{s})=\left\{\begin{array}{r}
\frac{\mathrm{p}}{1-\mathrm{p}\left(\operatorname{rmod} \frac{1}{\mathrm{p}}\right)} \\
* \frac{\text { residual energy of a node } * \mathrm{k}_{\mathrm{opt}}}{\text { average energy of the network }}
\end{array}\right.
$$

\subsection{IDEEC}

Improved Distributed Energy-Efficient Clustering Protocol (IDEEC)[14,15] is similar to DEEC; only difference is scaling factor which means the simplification power $\epsilon_{\mathrm{fs}}$ is compact by factor 10 . The scaling factor could be measured using following equation:

$$
\begin{aligned}
& \text { Scaling factor }=\{\operatorname{rand}() \\
& \left.\times \frac{\text { Area of Network field }}{\text { area of the cluster } \times \text { no. of nodes in a cluster }}\right\}
\end{aligned}
$$

And the possibility of knob should develop the cluster head using equation:

$$
\begin{aligned}
& P_{i} \\
& =\frac{\text { Energy of the } i \text { th node }}{\sum_{i=1}^{n} \text { Total energy of all the nodes within a cluster }}
\end{aligned}
$$

Threshold value will decide whether the particular knob will become the cluster head or not.

$$
\begin{aligned}
& \mathrm{T}\left(\mathrm{s}_{\mathrm{i}}\right) \\
& = \begin{cases}\frac{\mathrm{P}_{\mathrm{i}}}{1-\mathrm{P}_{\mathrm{i}}\left(\operatorname{rmod} \frac{\mathrm{P}_{\mathrm{i}}}{\sum_{\mathrm{i}=1}^{\mathrm{n}} \mathrm{P}_{\mathrm{i}}}\right)} & \text { if } \mathrm{S}_{\mathrm{i}} \in \mathrm{G} \\
0 & \text { otherwise }\end{cases}
\end{aligned}
$$

\section{SIMULATIONS AND DISCUSSIONS}

In this section, we simulate different clustering protocols in heterogeneous WSN using MATLAB and for simulations we use 100 nodes randomly placed in a field of dimension $100 \mathrm{~m} \times 100 \mathrm{~m}$. For simplicity, we consider all nodes are either fixed or micro-mobile and ignore energy loss due to signal collision and interference between signals of different nodes that are due to dynamic random channel conditions. In this scenario, we are considering that, BS is placed at center of the network field. We simulate DEEC, DDEEC, EDEEC, TDEEC and IDEEC for multi-level heterogeneous WSNs. Scenarios describe values for number of nodes dead in first, tenth and last rounds as well as values for the packets sent to $\mathrm{BS}$ by $\mathrm{CH}$ at different values of parameters $\mathrm{m}, \mathrm{m}_{\mathrm{o}}, \mathrm{a}$ and $\mathrm{b}$. we also examined these protocols in terms of overhead, end to end delay, packet delivery ratio and throughput. These values are examined for DEEC, DDEEC, EDEEC, TDEEC and IDEEC.

In heterogeneous WSN, we use radio parameters mentioned in Table 1 for different protocols deployed in 
WSN and estimate the performance for three level heterogeneous WSNs. Parameter $m$ refers to fraction of advanced nodes containing extra amount of energy a in network whereas, $m_{o}$ is a factor that refers to fraction of super nodes containing extra amount of energy $b$ in the network.

\section{Formulas for simulation parameters:}

1. Dead nodes: it is the nodes which died until last round, so, good performance which obtain minimum dead nodes.

2. Alive nodes: it is the nodes which still until the last round. Better performance with the higher alive nodes.

3. Packets sent to the BS: indicate quantity of packets received by $\mathrm{BS}$ for each round.

4. $\mathrm{CH}$ packets: indicate the quantity of sent packets to BS for each round.

5. Network Delay: This performance metric is used to measure the average end-to-end delay of data packet transmission. The end-to-end delay implies the average time taken between a packet initially sent by the source, and the time for successfully receiving the message at the destination. Measuring this delay takes into account the queuing and the propagation delay of the packets.

6. Overhead: it is the total number of packets are to be transferred or transmitted from one node to another. It includes the overhead of routing process, routing table and packet preparation in a sensor node.

7. Packet Delivery Ratio (PDR): can be measured as the ratio of number of packets delivered in total to the total number of packets sent from source node to destination node in the network. It is desired that maximum number of data packets has to be reached to the destination. As the value of PDR increases the performance of the network also increases.

8. Network Throughput: The end-to-end network throughput measures the number of packets per second received at the destination. It is considered here as an external measure of the effectiveness of a protocol. For good designed network the value should be high [17].
TABLE I

VALUE OF PARAMETERS

\begin{tabular}{|l|l|}
\hline Parameters & Values \\
\hline Network field & $100 \mathrm{~m}, 100 \mathrm{~m}$ \\
\hline Number of nodes & 100 \\
\hline$E_{o}$ (initial energy of normal nodes) & $0.5 \mathrm{~J}$ \\
\hline Message size & $4000 \mathrm{bits}$ \\
\hline$E_{\text {elec }}$ & $50 \mathrm{~nJ} / \mathrm{bit}$ \\
\hline$E_{f s}$ & $10 \mathrm{~nJ} / \mathrm{bit} / \mathrm{m} 2$ \\
\hline$E_{\text {amp }}$ & $0.0013 \mathrm{pJ} / \mathrm{bit} / \mathrm{m} 4$ \\
\hline$E D A$ & $5 \mathrm{~nJ} / \mathrm{bit} /$ signal \\
\hline$d_{o}$ (threshold distance) & $70 \mathrm{~m}$ \\
\hline$P_{\mathrm{opt}}$ & 0.1 \\
\hline
\end{tabular}

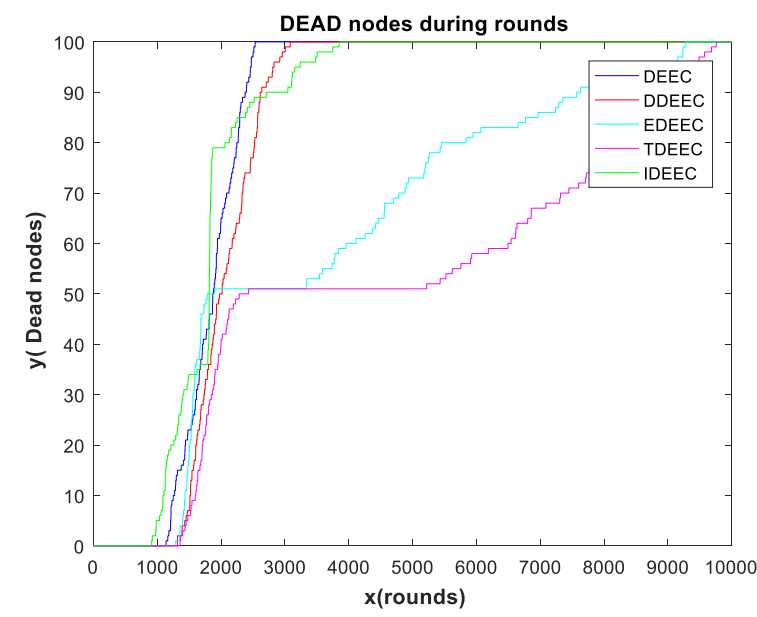

Fig. 1, Shows dead nodes number

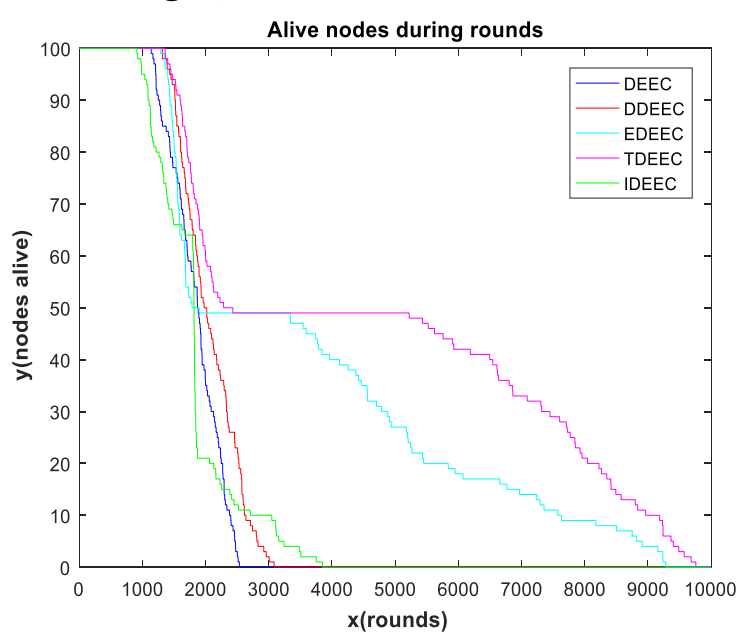

Fig. 2, Depicts Nodes alive during rounds 


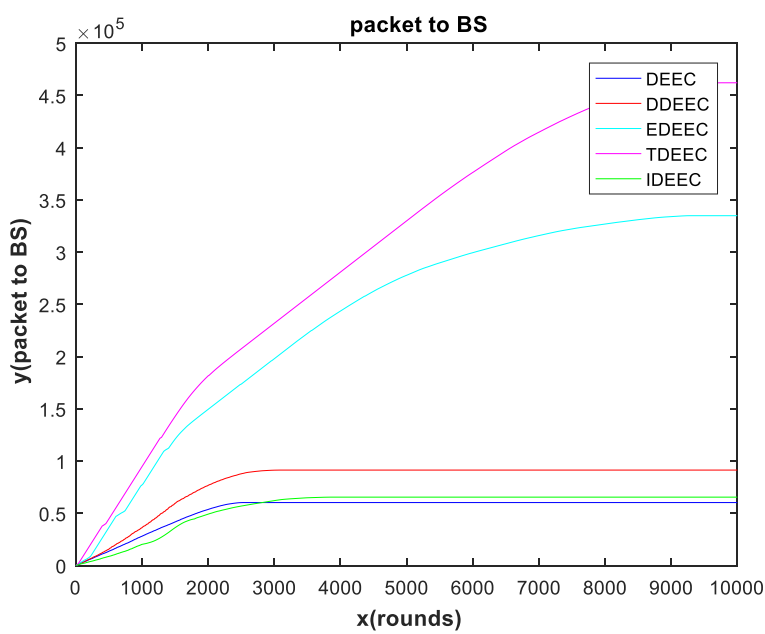

Fig. 3, Shows Packets sent to the BS

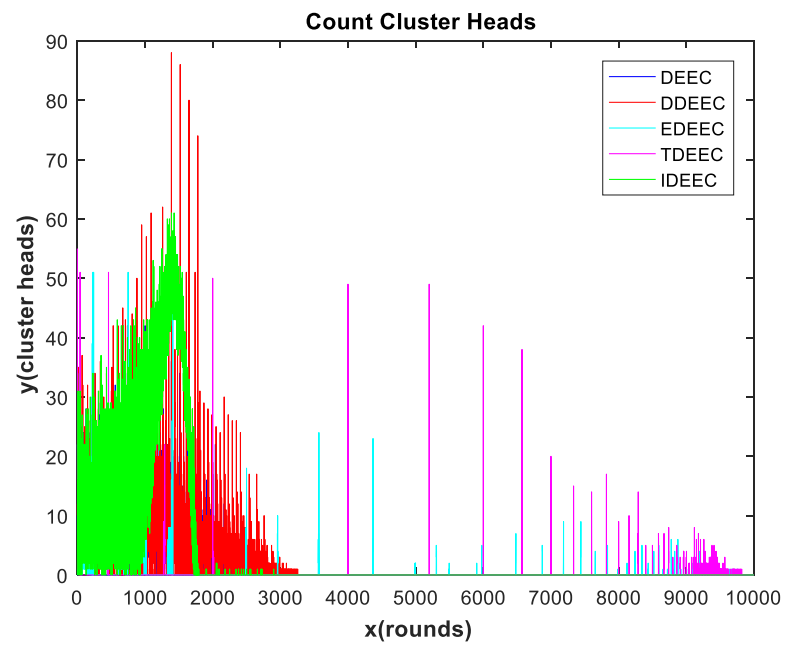

Fig. 4, Depicts Count Cluster Heads

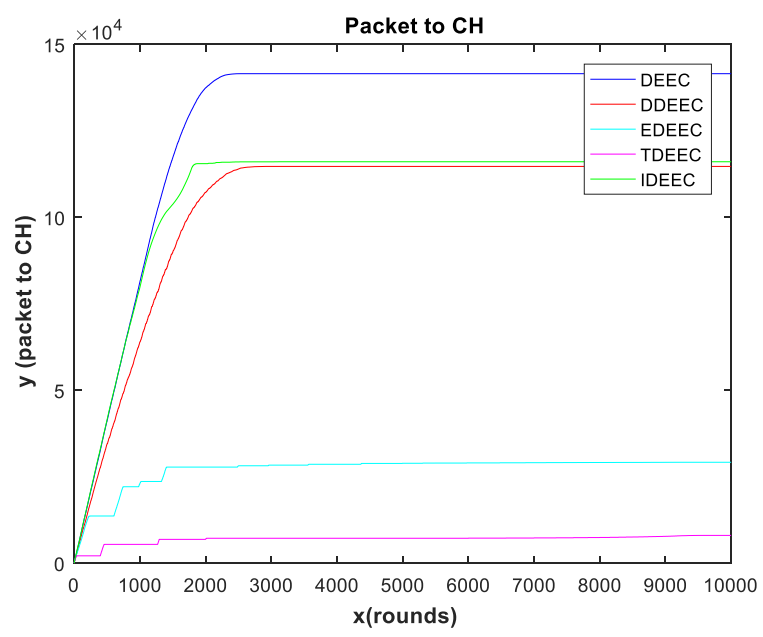

Fig. 5, Shows Packets sent to Cluster Head

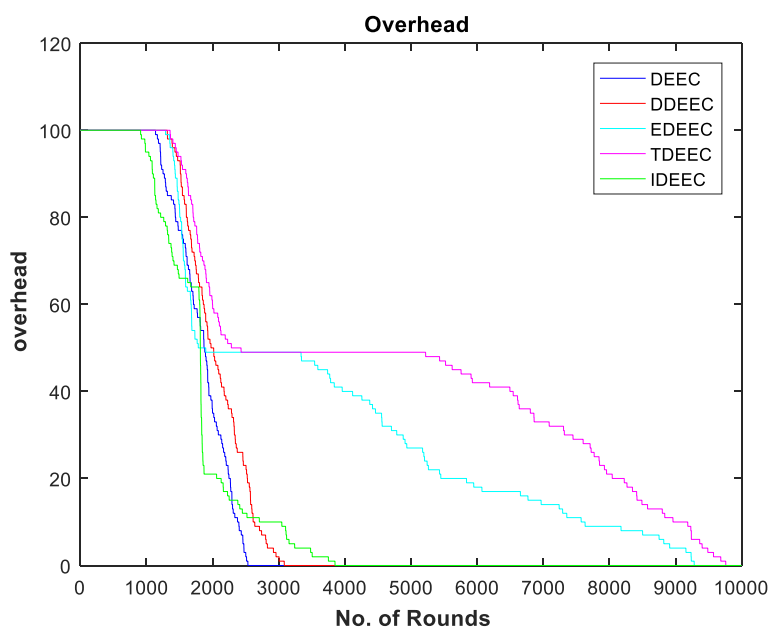

Fig. 6, Depicts Overhead of protocols

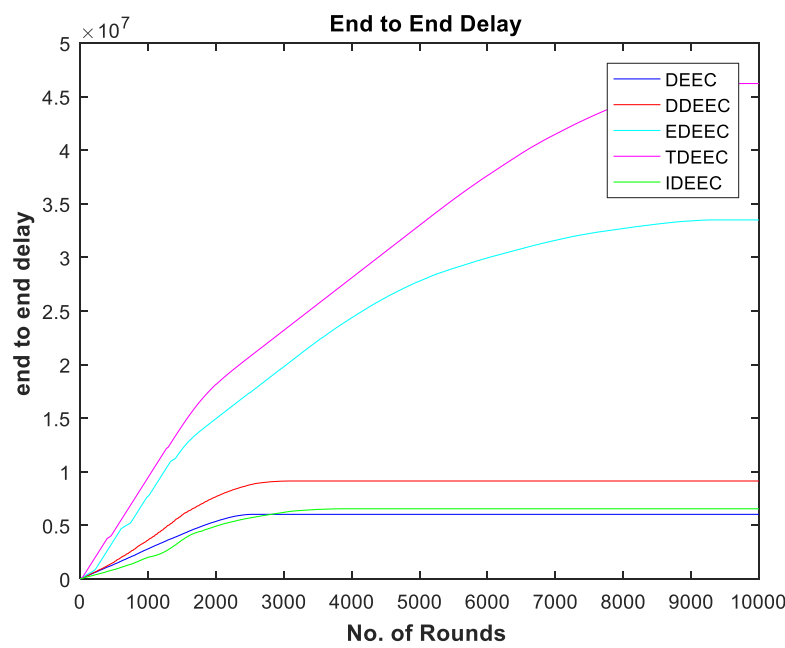

Fig. 7, Shows the End-to-End Delay.

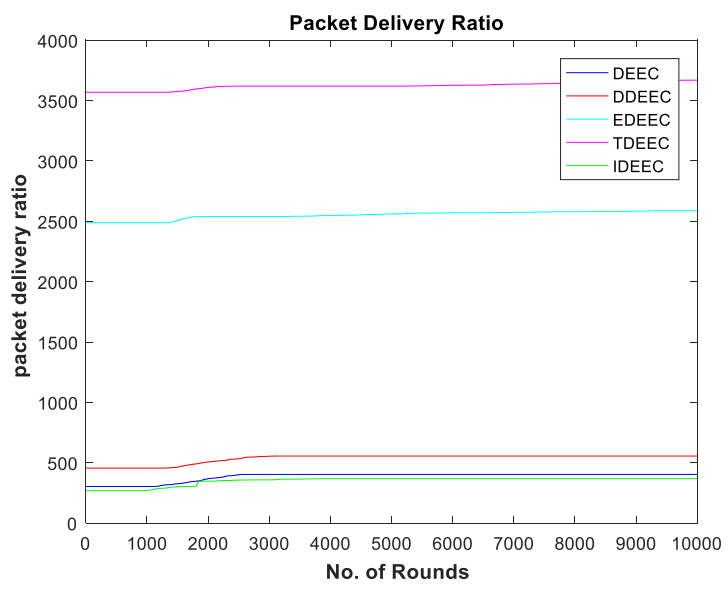

Fig. 8, Shows the Packet Delivery Ratio. 


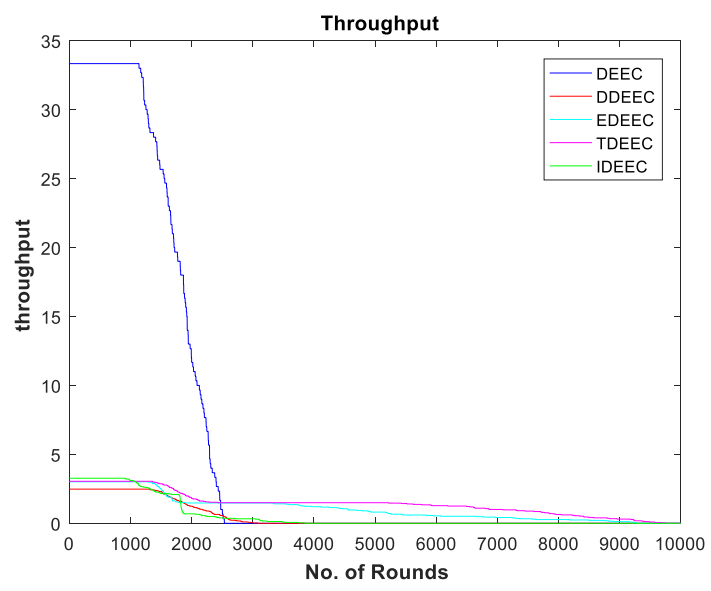

Fig. 9, Depicts the Throughput.

From Fig. 1 and 2, we examine that first node for DEEC, DDEEC, EDEEC, TDEEC and IDEEC dies at 1031,1208,1278, 1363 and 912 rounds respectively. Tenth node dies at 1280,1503,1429, 1568 and 1092 rounds respectively. All nodes are dead at $2817,2970,9207,9953$ and 3852 rounds respectively. It is obvious from the results of all protocols that in terms of stability period, TDEEC performs best of all; EDEEC performs better than DEEC, DDEEC and IDEEC but has less performance than TDEEC. DDEEC only performs well as compared to DEEC and DEEC has least performance than all the protocols. Stability period of DEEC, DDEEC and IDEEC is lower than EDEEC and TDEEC because the probabilities in TDEEC and EDEEC are defined separately for normal, advanced and super nodes whereas, DEEC, DDEEC and IDEEC do not use different probabilities for normal, advanced and super nodes so their performance is lower than EDEEC and TDEEC. However, instability period of EDEEC and TDEEC is much larger than DEEC, DDEEC and IDEEC. The number of nodes alive in TDEEC is quite larger than EDEEC because in TDEEC the formula of threshold used by nodes for $\mathrm{CH}$ election is modified by including residual and average energy of that round. So, nodes having high energy will become CHs.

In Fig. 3, shows that the package reaches with TDEEC and EDEEC protocols to base station performing better than DEEC, DDEEC and IDEEC protocols but TDEEC performance is very good other than protocols and the package arrives with excellent performance protocol and reaches the maximum with increased number of rounds.

Fig. 4, depicts that the cluster heads number in all protocols are decreased with increasing number of rounds. TDEEC, DEEC and EDEEC still for along rounds other than protocols.

DDEEC and IDEEC has high number of cluster heads in the beginning rounds but don't still for long rounds.

Fig. 5, depicts that the packet sends to Cluster head, DEEC, IDEEC and DDEEC are perfect than EDEEC and TDEEC performance; DEEC has the best performing other than all protocols; and the packet sends with TDEEC protocol is the worst performing in all number of rounds.

In Fig. 6, TDEEC method performed better than the other methods according to network overhead and was found to end after approximately more than 10000 rounds.

In Fig. 7, Shows that the End-to-End Delay for DEEC protocol has the best performance than other protocols; TDEEC protocol has the least performance. EDEEC, IDEEC and DDEEC protocols are in the middle.

In Fig. 8, we can see obviously that TDEEC and EDEEC protocols are the best in Packet Delivery Ratio; but TDEEC performs better with more than 3500 packet delivery ratio. IDEEC has the least performance.

In Fig. 9, TDEEC method performed better than the other methods according to network throughput and was found to end after approximately 9848 rounds.

\section{CONCLUSION}

We have examined DEEC, E-DEEC, T-DEEC, DDEEC and IDEEC for heterogeneous WSNs. Simulations prove that DEEC, DDEEC and IDEEC perform well in the networks containing high energy difference between normal, advanced and super nodes. Whereas, we find out that EDEEC and TDEEC perform well in all scenarios. TDEEC has best performance in terms of stability period and life time but instability period of EDEEC and TDEEC is very large. So, EDEEC and TDEEC are improved in terms of stability period while compromising on lifetime.

We proposed TDEEC (Threshold Distributed Energy Efficient Clustering) protocol which improves stability and energy efficient property of the heterogeneous wireless sensor network and hence increases the lifetime. Simulation results show that TDEEC performs better as compared to DEEC, DDEEC, EDEEC and IDEEC in heterogeneous environment for wireless sensor networks.

\section{Future Contribution}

In future many other techniques should be developed which should focus on improving the network performance so that the lifetime as well as the performance of the wireless senor networks can be improved.

Further research can be done on the above-mentioned issue.

\section{REFERENCES}

\section{Journal Papers:}

[1] I.F. Akyildiz, W. Su, Y. Sankarasubramaniam, E. Cayirci, "Wireless sensor networks: a survey, Computer Networks", 38 (4) (2002) 393-422. 
[2] P. Krishna, N.H. Vaidya, M. Chatterjee, D. Pradhan, "A cluster-based approach for routing in dynamic networks, ACM SIGCOMM Computer Communication Review", 27 (2) (1997) 49-65.

[3] B. McDonald, T. Znati, "Design and performance of a distributed dynamic clustering algorithm for Ad-Hoc networks", in: Proceedings of the Annual Simulation Symposium, 2001.

[4] V. Mhatre, C. Rosenberg, D. Kofman, R. Mazumdar, N. Shroff, "Design of surveillance sensor grids with a lifetime constraint", in: lst European Workshop on Wireless Sensor Networks (EWSN), Berlin, January 2004.

[5] W.R. Heinzelman, A.P. Chandrakasan, H. Balakrishnan, "Energy efficient communication protocol for wireless micro sensor networks", in: Proceedings of the 33 rd Hawaii International Conference on System Sciences (HICSS-33), January 2000

[6] W.R. Heinzelman, A.P. Chandrakasan, H. Balakrishnan, "An applicationspecific protocol architecture for wireless microsensor networks", IEEE Transactions on Wireless Communications 1 (4) (2002) 660-670.

[7] S. Lindsey, C.S. Raghavenda, PEGASIS: "power efficient gathering in sensor information systems", in: Proceeding of the IEEE Aerospace Conference, Big Sky, Montana, March 2002.

[8] O. Younis, S. Fahmy, HEED: "A hybrid, energyefficient, distributed clustering approach for ad hoc sensor networks", IEEE Transactions on Mobile Computing 3(4)(2004)660 - 669.

[9] G. Smaragdakis, I. Matta, A. Bestavros, "SEP: A Stable Election Protocol for clustered heterogeneous wireless sensor network", in: Second International Workshop on Sensor and Actor Network Protocols and Applications (SANPA 2004), 2004

[10]L. Qing, Q. Zhu, M. Wang, "Design of a distributed energy-efficient clustering algorithm for heterogeneous wireless sensor network", ELSEVIER, Computer Communications 29,2006, pp 2230- 2237.

[11] Elbhiri, B. , Saadane, R. , El Fkihi, S. Aboutajdine, D. "Developed Distributed EnergyEfficient Clustering (DDEEC) for heterogeneous wireless sensor networks", in: 5th International Symposium on I/V Communications and Mobile Network (ISVC), 2010.

[12] Parul Saini, Ajay.K.Sharma, "E-DEECEnhanced Distributed Energy Efficient Clustering Scheme for heterogeneous WSN", in: 2010lst International Conference on Parallel, Distributed and Grid Computing (PDGC - 2010).

[13] Parul Saini, Ajay.K.Sharma, "Energy Efficient Scheme for Clustering Protocol Prolonging the Lifetime of Heterogeneous Wireless Sensor Networks", International Journal of Computer Applications (09758887), Volume 6 No.2, September 2010 .

[14] Sahar Alsafi, Samani A. Talab, "Implementation of DEEC, DDEEC, EDEEC andTDEEC Protocols using MATLAB in WirelessSensor Network", Int. J. Advanced Networking and Applications, Volume: 12 Issue: 03 Pages: 45964600(2020) ISSN: 0975-0290.

[15] Benyin, X., Chaowei, W.," An improved distributedenergy efficient clustering algorithm for heterogeneous WSNs". In: IEEE Wireless Communications and Networking Conference (WCNC), pp. 19-22, San Francisco, CA (2017).

[16] Trupti Mayee Behera, Umesh Chandra Samal, Sushanta Kumar Mohapatra," Energy-efficient modified LEACH protocol for IoT application", IET Wirel. Sens. Syst. (C) The Institution of Engineering and Technology 2018, May 2018.

[17] Hamdy H. Hayed,Han ShabanHassan,'Performance Comparison of Various HierarchicalWSNRouting Protocols', Int. J. Advanced Networking andApplications, Volume: 11 Issue: 02 pp. 4218-4223, ISSN:09750290, 2019. 